\title{
La santé, « nouvelle frontière » de l'intégration européenne
}

\author{
Plaidoyer pour une Europe de la santé
}

Thomas Kostera

\begin{abstract}
Keywords : European integration, Europeanization, healthcare, patient mobility, solidarity, social citizenship

The article sets out the possible impact and the consequences that a growing Europeanization of national healthsare systems will have for European integration once a decision has been made for a real European approach to bealthcare. The article does not try to show how and why certain political tensions have emerged between the European and the national level concerning the nascent Europeanization of the healthcare sector. It rather imagines a step abead, the "new frontier" of European integration: it is a plea for a coberent and sustainable European approach to bealthcare wbich would embody a systematic and practical exploration of a Europe-wide notion of solidarity.

Mots clés : Intégration européenne, européanisation, soins de santé, mobilité des patients, solidarité, citoyenneté sociale

Cet article cherche à démontrer l'impact que peut avoir l'européanisation naissante des systèmes de santé nationaux - ainsi que ses conséquences sur l'intégration européenne d'une manière générale - à compter du moment où l'on ferait le choix résolu d'avancer vers une Europe de la santé, véritable et approfondie. Cet article ne vise pas à montrer ni comment, ni pourquoi certaines tensions sont nées entre l'Union européenne et le national, du fait de l'européanisation embryonnaire du secteur sanitaire, mais d'imaginer l'étape d'après, la «nouvelle frontière ». À ce titre, il s'agit d'un plaidoyer pour une Europe de la santé cohérente et durable, signe de l'exploration méthodique et pratique du principe de solidarité à l'échelle du « vieux continent ».
\end{abstract}




\section{Introduction}

Partons d'un présupposé théorique. Et si c'était la santé ? Et si la relance de l'intégration européenne passait par la mise en place de vraies politiques publiques en matière de santé ? Alors, que se passerait-il ? Quels changements cela occasionnerait-il pour les systèmes de santé nationaux des vingt-sept États-membres ? L'Union européenne (UE) a elle-même identifié plusieurs domaines d'avenir où sa valeur ajoutée pourrait apparaître bientôt (environnement et changement climatique, énergie, politique étrangère, etc.) et la placer au rang des " grandes puissances ». Certes, tous ces domaines sont très «vendeurs" et serviraient aisément de porte-étendards d'une nouvelle "grande Europe, mais tous ne connaissent pas le même avancement. Tous ne sont pas liés aux politiques extérieures en tant que tels, mais tous sont « visibles » de l'extérieur. Il s'agit d'avancées certainement très nécessaires, mais une question subsiste, à laquelle aucun de ces domaines ne permet de répondre : comment rapprocher les citoyens de l'intégration européenne ; comment leur montrer la nécessité de poursuivre ce rapprochement d'États-nations qui ne va pas de soi au regard de l'histoire du continent européen de ces derniers siècles? L'épisode du rejet du Traité établissant une Constitution pour l'Europe a montré qu'identification des citoyens à l'Europe et approfondissement de l'intégration sur un plan institutionnel n'étaient pas concomitants, que les institutions n'étaient pas des faits tangibles et que l'optimisation de leur organisation ne conduirait pas à un enthousiasme retrouvé, à un sentiment d'appartenance. Il faudrait donc construire une Europe plus concrète. Il faudrait surtout s'ingénier à bâtir un cadre de solidarité au niveau supranational et retrouver un liant sociopolitique à ce niveau.

C'est pourquoi la santé peut être la «nouvelle frontière » de l'intégration européenne, c'est-à-dire cette terre inexplorée qui devient le centre d'attention de chacun, en raison de son caractère essentiel. Par tribut à John F. Kennedy et à son programme électoral de 1960, c'est par le progrès de l'intégration dans cet espace de solidarité que pourra se vaincre l'état de déréliction dans lequel se trouve la construction européenne en ce début de $\mathrm{XXI}^{\mathrm{e}}$ siècle. Une "nouvelle frontière ", pourquoi ? Parce que la santé a ceci de spécifique qu'elle a à la fois trait au particulier - car touchant à la vie, etc. - et à l'universel, mais qu'elle demeure également un secteur économique et de ce point de vue une part importante du Produit intérieur brut (PIB) des pays de l'Organisation de Coopération et de développement économiques (OCDE). À travers les politiques publiques de santé, on touche donc à l'esprit de solidarité, tout en dynamisant notre économie (health is wealth). L'impact de l'UE sur les systèmes de santé, l'européanisation et ses conséquences au niveau national, commencent à être des sujets d'étude. Ici, nous tenterons très immodestement de démontrer l'impact que peut avoir l'européanisation naissante et celui qu'il pourrait avoir sur l'intégration européenne, à compter du moment où l'on ferait le choix résolu d'avancer vers une Europe de la santé ; où l'on orienterait délibérément l'intégration européenne vers un nouveau processus mettant la solidarité au centre. Ici, nous ne cherchons plus à montrer ni comment, ni pourquoi 
certaines tensions sont nées entre l'UE et le national, du fait de l'européanisation embryonnaire du secteur sanitaire, mais d'imaginer l'étape d'après, la « nouvelle frontière ».

La première partie de l'article se focalise sur le processus d'intégration européenne et sur les tensions qu'il fait peser sur les systèmes de santé des vingt-sept États-membres de l'UE. Alors que le premier chapitre brosse à grands traits l'évolution du droit primaire (les fameux Traités communautaires) et sa progressive prise en compte du secteur de la santé, le deuxième chapitre s'attarde sur les spécificités sociales et économiques des services de santé, mettant en exergue les valeurs quintessentielles des systèmes nationaux de santé. La traduction concrète du principe de solidarité, qu'ils institutionnalisent dans des cadres organisationnels et philosophiques différents est décrite dans le chapitre suivant. Depuis les années 1990, les systèmes de santé, sont devenus de plus en plus complexes dans leurs structures, suite à de multiples réformes. Réduction des coûts, tickets modérateurs pour certains médicaments ou traitements, listes d'attente pour certaines opérations sont quelques-unes des conséquences les plus visibles de ces changements d'ampleur. Dans certains cas, il est donc devenu plus « attrayant » pour un patient de profiter de l'offre d'un fournisseur de soins de santé d'un autre État-membre européen. Cette mobilité des patients est traitée dans le quatrième chapitre. D'apparence bénigne aux non initiés - ou à ceux qui ne retiennent que la faible proportion de citoyens apparemment concernés par cette dynamique -, elle met en réalité en question la régulation nationale des systèmes de santé dans des proportions non négligeables. Dans un seul et même mouvement, elle offre cependant aussi la possibilité de l'émergence d'un véritable espace européen de santé.

C'est pour la formation de celui-ci que plaide la seconde partie, en proposant d'explorer de possibles voies vers cette «nouvelle frontière » de l'intégration européenne. Dans une réflexion utopiste insistant sur la nécessité d'établir une solidarité entre les citoyens européens en matière de santé, le premier chapitre suggère la création d'une assurance maladie universelle de base au niveau européen. Elle assurerait le remboursement des soins primaires, grâce à un transfert d'une partie de l'imposition au niveau supranational. Les deuxième et troisième chapitres proposent la création d'une Agence européenne d'évaluation des technologies de santé (EAHTA) et d'une Agence européenne de détermination des prix et des taux de remboursements. Conçues comme prenant place dans un ensemble cohérent et complétant l'action de la Caisse d'assurance maladie européenne de base, elles permettraient d'asseoir ce nouveau dispositif échafaudé au niveau supranational. Enfin, les deux derniers chapitres proposent la création d'une véritable Direction générale de la santé publique au sein de la Commission européenne et l'extension des compétences communautaires en matière de santé. 


\section{La santé et l'intégration européenne, insubmersibles différences entre États ?}

\subsection{Le marché intérieur européen et la santé}

La santé a ceci de particulier qu'elle n'est pas à mettre au rang des politiques publiques les plus intégrées au niveau de l'Union européenne. C'est d'ailleurs un des domaines dans lequels la compétence supranationale de l'UE est la plus restreinte. Au sortir de la Seconde guerre mondiale, on la considérait davantage à travers le prisme d'une action sanitaire focalisée sur les maladies épidémiques, plaies des sociétés européennes de marchands du Moyen-Âge au XIX ${ }^{\mathrm{e}}$ siècle. Néanmoins, le «cadre d'approche géographique » était soit trop large (Organisation mondiale de la santé), soit trop restrictif (Benelux, Conseil nordique). «La réunion d'un certain nombre d'États européens relativement homogènes [étant] la condition sine qua non d'une action sanitaire unifiée » (Cassan 1989, p. 12), le cadre communautaire a pu apparaître comme un niveau d'approche légitime, auquel ces derniers ont consenti une part - infime - de leur souveraineté. Un des avantages de cette approche communautaire réside, entre autres, dans ce qu'une « action dans ce secteur serait des plus populaires et permettrait une collaboration plus étroite des peuples à l'établissement progressif d'une "Europe unie" » (ibid., p. 18).

Cependant, force est de reconnaître que peu a été fait pour la mise en commun du secteur de la santé au niveau communautaire. Le Traité de Rome ne reprend d'ailleurs pas l'avant-projet de Communauté européenne (1952) de Paul Ribeyre, le ministre français de la Santé de l'époque. «Ce texte visait à réaliser, en plus d'un marché commun des professions et des produits de santé, une très large coopération dans les domaines de la formation, de la recherche et de l'action sanitaire, ainsi qu'une unification progressive des régimes de Sécurité sociale »(Dubouis\&Blumann 2004, p. 147). La santé est donc absente du Traité de Rome (1957), à l'exception de certaines dispositions permettant de déroger aux règles régissant le marché commun, en matière de libre circulation des marchandises (Article $30 \mathrm{CE}$ ), de libre prestation des services ou de liberté d'établissement (Art. $46 \mathrm{CE}$ ). Sa protection relève alors de la compétence des États, principe originaire toujours en vigueur aujourd'hui et ayant notamment pour conséquence le maintien du vote à l'unanimité au Conseil de l'UE.

L’Acte Unique européen (1986) fait néanmoins "entrer la santé dans le marché intérieur", en permettant de prendre en compte son «niveau de protection élevé » (Traité CE, art. 95) dans l'édiction de normes communautaires conduisant à «l'établissement et [au] fonctionnement du marché commun » (ibid.). Pour ce faire, l'utilisation de la base juridique du nouvel art. 95 laisse entrevoir l'adoption de ces normes à la majorité qualifiée. Ce n'est qu'en 1992, avec la ratification du Traité sur l'UE, que la Communauté se dote d'un titre (XII) relatif à la "Santé publique". Là encore, la spécificité du secteur 
concerné contraint à la prudence et à des avancées mesurées. Ainsi, le seul article de ce titre, l'article $152 \mathrm{CE}$, établit que «l'action de la Communauté complète [simplement] les politiques nationales » (Traité CE, art. 152), qu’elle appuie, «si nécessaire » (ibid.), leur action et qu'elle « respecte pleinement les responsabilités des États-membres en matière d'organisation et de fourniture de services de santé et de soins médicaux » (ibid.). Par conséquent, les États-membres gardent la responsabilité principale en matière de santé. L'Union européenne ne peut intervenir que pour compléter les actions des États-membres et les faire converger vers des objectifs communs. Santé et protection sociale sont donc à placer parmi ces secteurs, de moins en moins nombreux désormais, où le principe de subsidiarité fait figure de règle indépassable. C'est, en effet, un secteur sensible et il sera, par la suite, expliqué pourquoi. Enfin, pour corroborer cette prise en compte très minimaliste dans le droit primaire européen, il est à noter que la santé est absente de l'article 2 CE concernant les "missions" attribuées à la Communauté européenne. L'action de celle-ci ne comporte qu'une "contribution à la réalisation d'un niveau élevé de protection de la santé » (Traité CE, art. 3). Ainsi, la santé occupe une place bien maigre au sein des "principes" (« Première partie » du Traité CE) de la Communauté.

\subsection{Les spécificités économiques et sociales du secteur de la santé}

Il va de soi que la santé a un cô̂t, un coût parfois exorbitant pour la collectivité. Pour autant, il est une acception non moins partagée et qui souligne la nécessité de ne pas envisager la question sous un angle purement économique : la santé n'a pas de prix. L'adage populaire ajouterait: "car la vie n'a pas de prix » (et le malade dépensera sans compter pour l'accès au traitement qui lui est nécessaire). Elle a, en effet, trait à la vie elle-même, puisque être en bonne ou mauvaise santé suppose déjà d'être, de vivre. Par conséquent «l'accès à des soins de santé de qualité peut s'avérer vital, au sens propre du terme»(Baeten 2004, p. 10). Administré à des organismes vivants, l'acte de soin entraîne alors la prise en compte des notions d'incertitude et de risque qui lui sont inhérents.

En matière de prestation de soins de santé, il est nécessaire de viser au consentement du destinataire - le patient. C'est ainsi ce rapport à l'éthique qui fait de la santé un secteur pas comme les autres. Nombreux y sont les sujets sensibles et à forte connotation morale : euthanasie, avortement, santé reproductive, pratiques quasi-eugéniques, acharnement thérapeutique, etc. Ces débats actuels sur les conditions d'exercice de la santé renvoient au plus profond des valeurs normatives et donc personnelles de chaque être humain, au rapport entre le bien et le mal dans les comportements humains. Ceci explique sûrement les fréquentes hésitations et les difficultés du politique à intervenir.

Ce qui fait la spécificité de la santé, c'est donc son rapport au particulier, à l'individu. Les praticiens le savent eux qui doivent sans cesse remettre en cause leur savoir, afin de 
l'adapter au patient qui consulte. Chaque cas est différent, chaque malade présente des pathologies propres et l'acte de soin est personnalisé. Les services de santé impliquent donc un rapport au personnel à un degré que les autres services connaissent assez peu. Cela signifie aussi que l'application de techniques de prestation fondées sur l'automaticité, la répétition - afin d'augmenter la productivité et l'efficience - trouvent ici peu leur place.

En outre, ce rapport subjectif au corps et à la sensation de bien-être psychique et physique entraîne une relation particulière entre patient et praticien. De plus en plus, ces derniers souhaitent pouvoir participer à la décision qui les concerne de manière intime. Cette revendication d'un accès démocratisé au savoir médical, cette volonté de comprendre et de se responsabiliser ne concerne pas tous les patients, mais elle participe d'un mouvement plus global des sociétés européennes individualistes. Toutefois, sa spécificité réside en ce qu'elle vise à atténuer cette situation de vulnérabilité du patient. Celui-ci n'est pas un consommateur comme les autres, il cherche à préserver un état de santé optimal, valeur suprême de nos sociétés, liberté fondamentale de l'être humain. Outre ce rapport au particulier, il faut considérer l'approche collective de la spécificité de la santé, la solidarité nécessitant l'organisation de systèmes à un niveau national d'ensemble.

Les sociétés humaines contemporaines accordent une valeur de premier ordre à la santé et valorisent un accès pour tous à des soins de qualité. L'idée de souffrance est devenue à ce point intolérable qu'une vie sans accès à des services de soins basiques n'est plus admise. À la base du raisonnement des États modernes, figure la nondiscrimination en matière d'âge, de genre et d'état socio-économique ou territoriale. L'action de l'État-providence et son développement reposent justement sur des valeurs de justice sociale, d'équité, d'universalité, de solidarité. C'est d'ailleurs ce qu'a rappelé le Conseil de l'UE, qui, lors de sa réunion des $1^{\text {er }}$ et 2 juin 2006, a mentionné ces valeurs communes des systèmes de santé de l'UE. Il y a ajouté six principes de fonctionnement communs : la qualité des soins, la sécurité du patient, des soins fondés sur des données probantes et sur l'éthique, la participation du patient, l'accès à la justice, le respect de la vie privée et la confidentialité (Conseil de l'Union européenne 2. juin 2006, p. 33-37). $\mathrm{Au}$ regard de ces valeurs et principes, il est possible d'avancer l'idée d'un modèle social européen, d'un "consensus européen », (Majnoni d’Intignano 2001, p. 40), fondé sur certaines zones de convergences entre États-membres, telles que l'existence, par exemple, d'une protection sociale universelle visant à prendre en charge la plus large partie de la population nationale. Il existe évidemment d'autres zones de convergence possible, telles que la qualité des services de santé notamment.

L'enjeu, à travers la mise en évidence de l'existence d'une spécificité de la santé, est, en réalité, la protection d'un modèle d'organisation du champ social au sens large, d'une vision européenne de la société, fondée sur des valeurs et des principes. Reconnaitre une spécificité comme vitale, c'est vouloir tout faire pour la préserver. Autrement dit, à travers la question des services de santé apparaissent les enjeux social (Sénat français, 
30 janvier 2007, p. 6) et politique. L'organisation de ces principes communs varie pourtant selon les États-membres. Elle est liée à leur constitution en tant qu'États-nations et aux traditions historiques et culturelles variées menant à la mise en place d'Étatsprovidence de la fin du XIX ${ }^{\mathrm{e}}$ au début du $\mathrm{XX}^{\mathrm{e}}$ siècle.

\subsection{Les différents systèmes de santé - une extrême hétérogénéité et complexité}

Une composante importante de l'État-nation en Europe est la solidarité entre citoyens. Celle-ci est un bien social, situé à équidistance entre la liberté et l'égalité des citoyens d'un même État. La solidarité désigne la mise en commun institutionnalisée des ressources individuelles, en vue de les utiliser à des fins communautaires. Cette mise en commun des ressources est la tâche d'un État-providence : il met en commun les ressources des citoyens afin de les protéger contre la pauvreté de vieillesse, contre les conséquences économiques d'une maladie ou du chômage. Par conséquent, l'Étatprovidence est la forme institutionnalisée de solidarité, qui doit à la fois être efficace et servir la justice sociale. La solidarité institutionnelle s'ajoute aux droits de participation politique et à d'autres éléments de l'identité nationale d'un État (Ferrara 2006, pp. 44ff). L'État-providence devient ainsi synonyme d'État-nation, car beaucoup de citoyens vont même considérer que la source de solidarité est la nationalité :

«It is generally true in sociology that the things which people believe are liable to be true in their consequences, and even if nationality is not based in any firm, objective truth, nationhood plays a major part in the formation of social policy [...]. The impact of nationality on contact, status and the structure of obligation tends to identify solidarity closely with national identity. Nationality defines the nation as the root of a solidaristic community » (Spicker, 2000, p. 53)

L'organisation de la solidarité par l'État-providence et leurs systèmes de santé peuvent varier considérablement d'un pays européen à un autre. En Europe, deux grands modèles d'organisation des systèmes de santé sont d'ordinaire mentionnés : le «beveridgien» et le «bismarckien» (cf. Esping-Anderson 1998). Dans les deux cas, l'assurance maladie collective et obligatoire fait partie de la protection sociale. Le modèle conçu par Lord William H. Beveridge (1879-1963), et présenté dans un rapport au Parlement britannique en 1942, promeut la création d'un service national de santé, de nature universelle et d'accès gratuit (pour les résidents). Il est financé en majeure partie par un impôt; la gestion du système en revient à l'État. «Les hôpitaux sont publics et les professionnels de santé sont des fonctionnaires salariés ou des professionnels indépendants sous contrat » (Polton 2005, p. 54). Créé en 1948, le National Health Service britannique (ou NHS) en est le parangon, les systèmes de santé des pays scandinaves appartenant également à ce modèle. À la différence du premier néanmoins, les seconds présentent des organisations beaucoup plus décentralisées. 
Le système bismarckien constitue un autre système, radicalement différent du premier car basé sur des solidarités professionnelles : c'est ainsi le fait d'être travailleur qui octroie une couverture sociale et l'assurance-maladie est financée par des cotisations. La grande différence de ces systèmes consiste en la séparation entre assurance maladie et organisation de la distribution de soins et on y retrouve une certaine intrication entre public/privé, secteur lucratif/non lucratif. Les services de santé ne sont donc pas uniformément rendus. Enfin, la solidarité au fondement du système est ici soumise à un certain éclatement entre les caisses, ce qui fait peser une tension sur sa vocation universelle. Ce deuxième grand modèle se retrouve évidemment en Allemagne, mais également dans le reste de l'Europe continentale, avec de larges variations entre les États de l'UE.

Comparer les systèmes de santé nécessite de prendre en compte plusieurs facteurs de leur architecture globale : «le mode de financement, le mode de gestion, les relations avec les structures et les professionnels, l'organisation de la production de soins et les filières de patients, le contrôle de l'accès aux soins spécialisés et/ou hospitalier » (ibid.). À la lumière de ces différents facteurs, de nombreuses variations sont possibles. C'est la raison pour laquelle il n'existe pas deux systèmes totalement identiques dans l'Europe des vingt-sept. C'est aussi une des raisons pour lesquelles vouloir identifier l'essence ultime des services de santé en Europe peut être extrêmement malaisé. Il n'en demeure pas moins une constante : les États-membres sont pleinement responsables de leur système de soins, les pouvoirs publics jouent un rôle fondamental de " pilotage » du système, afin de permettre l'accès à tous à des soins de qualité (Baeten 2004).

Après la fin des «Trente Glorieuses » de l'État-providence et la crise économique des années 1980, la plupart des pays européens a entamé des réformes structurelles de leurs États-providence dans une volonté de réduction des coûts. Les systèmes de santé n'échappaient pas à ces réformes : «Ils ont fait, dans la décennie 1990, l'objet d'une intense activité réformatrice, avec des objectifs de meilleur contrôle des coûts ou d'amélioration de l'efficience. Ces réformes ont souvent eu un caractère structurel, visant à modifier en profondeur l'architecture et le mode de gestion hérités de l'histoire. » (Polton 2005, p. 56).

Dans une analyse quantitative et qualitative, Rothgang et al. (2008) ont analysé les changements structurels des systèmes de santé pendant les deux dernières décennies. Ils ont ainsi trouvé que la plupart des pays occidentaux ont accru le rôle du financement privé des soins de santé. Ce rôle accru se traduit par exemple soit par l'introduction de tickets modérateurs pour certains médicaments ou traitements, soit par une baisse des remboursements des soins par l'assurance maladie. Parallèlement, le montant du financement public des systèmes de santé, mesuré en pourcentage du PIB, a augmenté également. On peut ainsi dire que, même si la part de financement privée des soins a augmenté, les systèmes de santé n'étaient pas privatisés au cours des dernières décennies. Pour ce qui est des prestations de soins de santé, Rothgang et al. ont montré que beaucoup de pays européens ont privatisé un certain nombre d'hôpitaux. En d'autres ter- 
mes, dans ces cas-ci, l'assurance maladie publique couvre toujours le traitement, mais les médecins ne sont plus employés par l'État et l’infrastructure nécessaire est gérée par des organismes privés. Cependant, la prestation privée de soins prévaut dans des systèmes bismarckiens, tandis que dans les systèmes beveridgiens, la prestation publique des soins de santé prévaut (Rothgang et al., 2008, pp. 136ff).

De surcroit, plusieurs pays européens expérimentent une déviation de la régulation publique traditionnelle de leur système de soins de santé. Ainsi, le National Health Service au Royaume-Uni était très centralisé. Au cours de ces dernières années, certaines compétences ont été déconcentrées et la marge de manœuvre des hôpitaux locaux quant aux capacités de certains traitements spécialisés a fortement augmenté. En Allemagne, c'est la tendance inverse qui a cours : certaines compétences de l'administration corporatiste bipartite ont été transférées vers l'État. Cela se manifeste dans le rôle accru de ce dernier dans la fixation des taux de cotisation. D'autres compétences ont été transférées au secteur privé : les assurances maladie en Allemagne peuvent agir comme un acteur privé sur le marché quand elles passent contrat avec des fournisseurs d'appareils médicaux. C'est pourquoi les vagues successives de régulation ont immanquablement conféré un caractère "hybride» aux systèmes de santé européens (ibid., pp. 138-146). Dès lors, ceux-ci s'éloignent lentement de leurs identités d'origine, « bismarckiennes » ou «beveridgiennes », ce qui les rend systèmes encore plus uniques et complexes.

Dans le même temps, ces systèmes doivent faire face à une influence croissante de l'UE. Si on garde présent à l'esprit l'importance du cadre national de l'État-providence dans la régulation du champ de la solidarité, il n'est pas surprenant que les Étatsmembres de l'UE considèrent que régulation, financement et prestation des soins de santé soient essentiellement des prérogatives nationales. Dès lors, leur reticence s'illustre à chaque velléité de transfert des compétences au niveau européen : «There is no such thing as a European healthcare system, and as long as decisions on financing, organization and service delivery are taken at a national level, there is little chance of one existing» (Steffen, Lamping \& Lehto, 2005, p. 3). Pourtant, la question sensible de la mobilité des patients au sein de l'UE pourrait conduire au dépassement d'une régulation nationale des politiques de santé.

\subsection{La mobilité des patients : vers le dépassement d'une régulation nationale des soins de santé ?}

L'article 152 CE établit que «l'action de la Communauté complète les politiques nationales », qu'elle appuie, «si nécessaire » leur action et qu'elle « respecte pleinement les responsabilités des États-membres en matière d'organisation et de fourniture de services de santé et de soins médicaux ». Il essaie de limiter chaque compétence de l'UE en matière de soins de santé, mais lui en laisse d'autres en santé publique, notamment la prévention de maladies. Pourtant, au niveau national, prestation des soins de santé et 
santé publique sont imbriquées, ne font qu'un. À l'échelon européen, la santé est alors une politique « divisée » (Harvey\&McHale 2004, p. 80). Toutefois, cette division est désormais mise en question par le phénomène de la mobilité des patients en Europe.

Celle-ci peut être définie comme "chaque voyage d'un patient d'un pays à un autre » (Baeten\&Glinos 2006, p. 18). Les patients peuvent avoir des motivations très variées pour quitter leur pays d'origine dans le but de chercher un traitement médical dans un autre pays de l'UE. Même si le patient «normal» voudrait être traité par un médecin qu'il connait, on peut identifier plusieurs facteurs qui motivent les patients à se déplacer dans un autre pays : l'existence d'un traitement spécialisé dans l'État d'accueil, des frais non remboursés considérables dans l'État d'origine, voire même une faible qualité des soins proposés dans l'État d'accueil. Le premier de ces facteurs semble être décisif pour les patients, car dans certain pays, un traitement pourrait s'avérer non disponible dans un délai de temps raisonnable. Il s'agit notamment des tristement célèbres listes d'attente pour interventions chirurgicales, dans les systèmes de santé beveridgiens. Parfois, c'est le traitement médical lui-même qui n'existe pas, obligeant le patient au déplacement (ibid., p. 6).

Les régions frontalières sont au cœur des réflexions à ce sujet : si un patient partage la même langue ou la même culture que les habitants d'un autre État-membre, il pourrait alors décider de s'y faire traiter, sans devoir voyager plus longuement dans l'État d'origine dont lequel il est ressortissant (ibid.). Le nombre de patients ayant effectivement bénéficié d'un traitement hors de leurs frontières est restreint et avoisinerait $1 \%$ de tous les patients. Cependant, ce taux peut s'élever jusqu’à 7\% dans des régions frontalières. Certains patients préfèrent se servir d'un intermédiaire professionnel qui va arranger le séjour dans l'hôpital d'un autre État-membre. Ces intermédiaires sont normalement sollicités par les patients lorsqu'existent des listes d'attente pour une opération ou lorsque le traitement ne serait pas couvert par l'assurance maladie du pays d'origine (ibid.).

Traditionnellement, l'accès aux soins dans un autre État-membre de l'UE était considéré à travers le prisme de la libre circulation des travailleurs et régi par le règlement $\mathrm{n}^{\circ} 1408 / 71$. Selon son article 22, tout individu couvert par le régime d'assurance maladie de son État membre ${ }^{13}$ pouvait obtenir la prise en charge de soins reçus en dehors de celui-ci. Lors d'un séjour temporaire à l'étranger, un patient sera pris en charge pour les soins urgents ("inopinés») uniquement; ceux pouvant attendre le retour dans l'État d'origine n'étant pas pris en charge. Pourtant, plusieurs patients qui ont déjà bénéficié de soins transfrontaliers non urgents, ont tout de même demandé le remboursement

${ }^{13}$ La Carte européenne d'assurance maladie (CEAM), qui a remplacé le formulaire E111 à partir du $1^{\text {er }}$ juin 2004, sert à prouver que le patient est bien couvert par son régime d'assurance maladie. 
des frais à leurs assurances maladies. Suite au refus de leurs assurances maladie de rembourser ces frais de traitement dans un autre État-membre, certains d'entre eux ont déposé un recours devant la Cour de justice des Communauté européennes (CJCE).

Dans son arrêt Kohll (CJCE 28 avril 1998), la Cour a instauré une seconde procédure d'accès aux soins à l'étranger. S’inscrivant dans la logique de la libre prestation de services prévue par le Traité, celle-ci pose davantage problème aux États-membres. En effet, le patient peut demander le remboursement de soins à l'étranger, comme si ceux-ci avaient été fournis sur le territoire national, sans attendre une hypothétique autorisation. Selon la Cour (CJCE, 23 octobre 2003), cette seconde procédure ne fait pas pour autant disparaître la première. De surcroît, le second aspect lié à la mobilité des patients est celui du remboursement des soins, qui met en évidence de grandes disparités dans les coûts des soins, selon les États-membres. Les facteurs de variation sont notamment l'utilisation de technologies plus ou moins avancées, la qualité des infrastructures et la rémunération des prestataires (Simon 2005, p. 173). Ces variations ont une conséquence pour le patient qui cherche à se faire rembourser la plus grande part des soins fournis. En effet, selon que les tarifs de l'État d'accueil sont inférieurs ou supérieurs à celui d'affiliation, le patient aura intérêt à opter pour la procédure ouverte par la jurisprudence Kohll (dans le premier cas), ou à obtenir l'autorisation préalable de son organisme de sécurité sociale (dans le second cas) (ibid. pp. 174, 175). En outre, la jurisprudence de la Cour a, depuis lors, amplifié les conséquences pour les patients et les systèmes de santé. Elle a ainsi assuré que les premiers devaient être garantis de recevoir un remboursement au minimum identique à celui qu'ils auraient eu dans leur État d'origine. Ils peuvent même, dans certains cas (soins hospitaliers), espérer bénéficier du meilleur tarif de remboursement, celui de l'État d'accueil ou celui de l'État d'affiliation » (CJCE 12 juillet 2001). La situation décrite précédemment vaut pour les soins programmés. Pour ce qui est des soins d'urgence (ou inopinés), l'article 22 précité permet à un patient d'obtenir un remboursement sur la base des tarifs de l'État d'accueil, mais l'éventualité d'un paiement complémentaire n'est pas garantie. La Cour n'ayant pas encore eu à connaitre d'une telle demande, il n'est donc pas possible de savoir si les règles de la libre prestation des services s'ajoutent à celles de l'article 22 (ibid.).

Un des points fondamentaux de l'arrêt Kohll réside dans le rappel par la Cour de l'application de la libre circulation aux systèmes de sécurité sociale. Le fait que les services de santé soient fournis dans le cadre de ces systèmes, qui interviennent pour le remboursement des soins, n'en empêche pas moins les articles 49 et suivants du traité de s'appliquer (ibid.). Une des conséquences immédiates qui en découle est que la délivrance obligatoire d'une autorisation préalable fonde une entrave à la libre prestation de services, interdite par l'article 49 CE (CJCE 28 avril 1998). La Cour accepte une telle entrave seulement dans certains cas : «Dès lors, pour qu'un régime d'autorisation administrative préalable soit justifié alors même qu'il déroge à une telle liberté fondamentale, il doit, en tout état de cause, être fondé sur des critères objectifs, non discriminatoires et connus à l'avance, de manière à encadrer l'exercice du pouvoir d'appréciation des auto- 
rités nationales afin que celui-ci ne soit pas exercé de manière arbitraire $[\ldots]$. Un tel régime d'autorisation administrative préalable doit de même reposer sur un système procédural aisément accessible et propre à garantir aux intéressés que leur demande sera traitée dans un délai raisonnable et avec objectivité et impartialité, d'éventuels refus d'autorisations devant en outre pouvoir être mis en cause dans le cadre d'un recours juridictionnel. » (CJCE, 12 juillet 2001)

Depuis 1998, la Cour a répété constamment le jugement Kohll, confirmant ainsi que les règles du Traité s'appliquent bien aux soins de santé. Autrement dit, peu importe que le patient paie de sa poche pour les soins qu'il reçoit, puis introduise une demande de remboursement auprès de son organisme référent, ou bien au contraire que le prestataire de soins reçoive directement le paiement de la part du fonds d'assurance maladie (ou du budget national) compétent (CJCE 13 mai 2003). La portée de la libre circulation s'étend donc même aux services médicaux prestés dans le cadre des systèmes nationaux de soins, financés directement par l'impôt. C’est ce que rappelle la Cour dans l'arrêt Watts : «Il a déjà été jugé qu'une prestation médicale ne perd pas sa qualification de prestation de services au sens de l'article $49 \mathrm{CE}$ au motif que le patient, après avoir rétribué le prestataire étranger pour les soins reçus, sollicite ultérieurement la prise en charge de ces soins par un service national de santé [...]» (CJCE 16 mai 2006).

Ces arrêts pourraient limiter dans l'avenir la marge de manœuvre nationale de contrôle des systèmes de santé. En outre ils «menacent» des bases traditionnelles des systèmes de santé : Les limites géographiques d'un système de santé correspondent normalement aux frontières de l'État-nation. Dès lors, un État-membre de l'UE est obligé de rembourser des soins médicaux non urgents sans autorisation préalable la conception nationale des soins de santé est mise en question (Lamping 2005: 31). En outre, le contrôle des États-membres sur les bénéficiaires peut fortement diminuer. Tandis que les États-membres avaient traditionnellement restreint l'accès aux soins non urgents pour leurs propres citoyens, ils doivent maintenant garantir un accès pour tous les citoyens de l'UE. Un État-membre pourrait être obligé d'accepter sur son propre territoire que le statut de bénéficiaire de l'assurance maladie d'un citoyen soit défini par un autre État-membre, c'est-à-dire qui remplit les critères pour avoir accès aux soins (ibid.). Les États-membres sont également confrontés à une certaine perte de contrôle de la consommation de soins de santé par les citoyens. Ce « découplage » peut aussi être le fait des prestataires de soins de santé et a trait à la qualité des soins de santé. Des médecins, pharmaciens et infirmières étrangers ne devraient plus se voir entravés d'offrir leurs services sur le marché des soins de santé d'un autre État-membre. Les systèmes de santé nationaux dans l'UE sont ainsi confrontés à un problème fondamental :

«By facilitating the free movement of patients, the ECJ subjects the national/insurance health systems to indirect competition amongst them, hence pushing them to rationalize and promote efficiency. At a time where all the systems of social insurance in Europe go through a profound crisis - for economic, 
demographic, political and other reasons - this choice of the ECJ may prove particularly burdensome, at least in the short-term » (Hatzopoulos 2008, p. 13).

Si les services de santé sont des services comme les autres au regard du droit communautaire, les conséquences pour les systèmes de soins qui les encadrent sont majeures. Il s'agit de garantir les mécanismes de la solidarité, une offre de soins de qualité à tous, tout en maintenant l'équilibre du système en général et notamment du financement de ces soins. Les États-membres sont donc contraints de s'adapter et de prendre en compte la dimension européenne dans leurs actions de régulation et de coordination :

«The development of the right to effective and speedy medical treatment by the Court of Justice has made it clear that the challenges that national healthcare system in all member states $[\ldots]$ are now facing and will face, cannot be solved by any member state alone, and that therefore new solutions at the European level must be found» (Kaczorowska, 2006, p. 369).

Par conséquent, la jurisprudence de la Cour sur la mobilité des patients pourrait avoir des effets déstabilisants pour les systèmes de santé des États-membres. Cependant, elle constitue aussi de nouveaux droits pour tous les patients européens, lesquels étaient auparavant soit cantonnés dans leur État d'affiliation, soit aisés et donc aptes à profiter de services plus performants dans d'autres pays, mais à leurs frais.

Les difficultés d'adaptation à la dimension européenne varient pour les Étatsmembres en fonction de l'organisation institutionnelle de leurs systèmes de santé. En théorie, quelques États-membres pourraient même gagner économiquement à la mobilité des patients. Par conséquent, les positions politiques quant à une nouvelle directive sur le sujet varient considérablement entre les États-membres :

«From an overall perspective, where member states differ is whether they believe that the case-law strikes the right balance between patients' rights and protecting the particularities of the national health systems or whether they argue that the case-law is formulated too much in favor of the internal market» (Sindbjerg Martinsen, 2007, p. 38).

Il semble que surtout les Pays-Bas et la Belgique soient des avocats des droits des patients. Selon les Pays-Bas, la mobilité des patients pourrait être bénéfique pour l'utilisation efficace de l'infrastructure des soins médicaux. En outre, la Belgique, l'Espagne, la Finlande et le Luxembourg ont souligné qu'il faudrait clarifier les dispositions légales pour les patients et qu'il faudrait accroitre la transparence. D'autres Étatsmembres, comme la Suède, l'Allemagne, le Danemark, la République Tchèque et le Royaume-Uni regrettent le manque de définition commune de ce qu'est un traitement médical dans un hôpital. Tandis que la CJCE avait confirmé le système d'autorisations préalables pour un traitement dans un hôpital, les patients n'en ont plus besoin pour le 
traitement chez un médecin. Le Danemark a surtout évoqué le fait qu'un traitement médical chez un médecin pourrait se révéler très onéreux dans le cas d'une imagerie par résonance magnétique fonctionnelle (IRM), par exemple. Par conséquent, ces Étatsmembres voudraient avoir la possibilité de soumettre leurs patients à une procédure d'autorisation pour certains soins chez un médecin (Sindbjerg Martinsen, 2007, pp. 39f). Tandis que les définitions préoccupent beaucoup les «anciens» Étatsmembres, les «nouveaux » États-membres ont des soucis très différents. À première vue, il semble qu'une mobilité des patients accrue pourrait être économiquement bénéfique, car les coûts de traitement sont environ $50 \%$ plus bas dans ces pays que dans les États-membres de «l'UE des 15 ». Mais le problème qui se pose pour ces pays concerne les capacités - l'entrée d'un grand nombre de patients étrangers pourrait entraîner des listes d'attente pour les citoyens du pays concerné. De plus, les pays comme la Lituanie, la Lettonie, l'Estonie, la Slovaquie et Malte s'inquiètent des coûts de traitement dans un autre pays, pour leurs propres citoyens. Dans l'hypothèse où un patient d'un nouvel État-membre profiterait de soins médicaux dans un ancien État-membre, les systèmes de santé des nouveaux États-membres devraient payer jusqu'à cinq fois le montant des coûts d'un traitement dans un nouvel État-membre (ibid., pp. 42f). Etant donné ces craintes, les États-membres auraient intérêt à renforcer leur coopération, mais ils essayent en même temps de limiter le nombre de possibilités de traitement à l'étranger pour les patients qui veulent quitter leur système national de santé. On pourrait alors en conclure que la seule solution résiderait dans l'émergence d'un véritable espace européen de santé :

«The judgments of the Court of Justice concerning the patients' rights to crossborder health and long-term care caused not only an increase of personal rights but even more a fundamental change in understanding what European health policy reciprocal to the European and national level in the future really could mean. [...] We will witness the emergence of a new European area of health. Systems, more often than not only judged according to their limited expenditure of public funds, will have to understand that civic participation in a new and enlarged health care market will turn patients to consumers and force paying bodies at a national level to act accordingly. Reaching out instead of fencing up might thus become a new and clearly enriched variant of subsidiary systems of health care » (Sieveking, 2007, p. 40). 


\section{Vers une Europe de la santé - ébauche d'un manifeste utopiste}

\subsection{Création d'une assurance maladie universelle de base}

Les développements et réflexions de cette partie partent d'une prémisse : les États providence européens, puissamment ancrés dans leur relativement longue tradition, tendent à imaginer leur réforme dans un cadre purement national. L'Europe est quasiment absente et c'est un tort, mais un tort qui s'explique aisément par la difficulté de penser le changement d'organisations complexes. C'est un tort, mais non rédhibitoire. Si l'organisation et la planification des soins - les aspects organisationnels et institutionnels - pourraient demeurer du ressort national (et régional), en fonction des nécessités variables des différents territoires de l'Union, il faut penser aux traductions concrètes d'une solidarité européenne. Il faut tenter, pour cela, d'avancer vers davantage de convergence des systèmes. Dans le contexte actuel d'explosion des dépenses de santé, les États-providence nationaux semblent en effet montrer leurs limites, ou du moins, leur difficulté à se réformer. Faire converger les systèmes nécessite une révolution sans doute progressive, mais que notre modèle utopiste verra comme abrupte - générale de la partie des systèmes de santé consacrée à l'évaluation des traitements et à leur remboursement.

Il faut tout d'abord se poser la question de la tendance actuelle. Les systèmes de santé convergent-ils en Europe ? A la lumière de la présentation synoptique précédente (cf. ci-dessus), il est aisé de douter d'un quelconque rapprochement entre des systèmes si opposés jusque dans leurs fondements. La réponse à la question précédente est, « sur le plan des principes : non. L'Union européenne n'harmonisera pas les siens, et rien ne tend à rapprocher les fondements philosophiques des [grands types de systèmes de santé qui s'y trouvent]. En Europe, la logique universelle, porteuse d'égalité et de justice sociale, gagne du terrain, alors que la logique professionnelle, porteuse d'inégalités et de corporatisme, en cède. La seconde domine pourtant dans les pays en développement où les travailleurs accèdent les premiers à une assurance maladie et où les systèmes universels sont synonymes de pauvres. La logique libérale, porteuse d'efficacité et d'innovation, se diffuse » (Majnoni d'Intignano, B., 2001, p.109). Ce dernier jugement, contestable au regard des constats énoncés précédemment, n'en révèle pas moins une tendance de fond des processus de réforme des systèmes de santé européen.

Il faut ensuite imaginer quelle pourrait être la meilleure manière de faire converger ces systèmes, tout en gardant à l'esprit l'objectif ultime de la régulation en matière de santé.

«La régulation est l'art de mettre au point et de régler un système complexe afin d'en assurer un fonctionnement satisfaisant. [C'est] l'art de répondre au double objectif suivant : d'une part, garantir un accès équitable à des soins de qualité, sans 
liste d'attente et, d'autre part, assurer l'efficience économique de l'ensemble du système, et donc éviter le gaspillage des ressources » (Mordelet P., 2006, p. 9).

Dans le système imaginé ici, l'Union européenne acquiert un rôle dans la seconde, car l'aspect planification / organisation des soins du système est laissé aux États-membres. Bien entendu, le séparer des fonctions de détermination des prix et de remboursement relève purement de la posture axiomatique. Les État-providence européens se sont bâtis progressivement en englobant ces fonctions, rendant difficile une conceptualisation différenciée.

Cependant, faire converger les systèmes de santé européens nécessite de prendre en considération certains préalables. En effet,

« dans le domaine de la santé, les orientations politiques dépendent des systèmes de valeurs et des choix de société. [...] La santé, c'est donc aussi la manière dont celle-ci est perçue par des individus malades. [...] La réforme du système de santé [...] doit prendre en compte ce débat sur les différentes conceptions de la santé [...] et sur la crise actuelle des valeurs. Le progrès médical est tel, de nos jours, que les capacités d'intervention sur la vie des individus à leur naissance (néonatologie) ou avant leur mort (réanimation) sont décuplées. Cette situation place les décideurs politiques dans l'obligation de faire des choix et de définir des priorités. [...] Ces choix reposent sur des valeurs éthiques et morales qui sont plus importantes que les arguments financiers et de régulation [...] Toute réforme [...] doit donc partir de cette réflexion et intégrer des valeurs éthiques comme la solidarité, la justice sociale et l'équité. [Si] les pays de l'Union européenne ont tous, aujourd'hui le même système de valeurs » (Ibid., 2006, p. 9-10),

au premier rang desquels ce sentiment de solidarité - nécessaire étai du ciment social -, ils semblent se refuser en revanche à l'étendre à un niveau supranational.

Cet article vise à les y pousser. Il s'agit non moins que de créer une assurance maladie de base, de type universelle. Cette Caisse d'assurance maladie transnationale assurerait le remboursement des soins primaires. Plus que de calquer un des systèmes préexistants, l'ambition conduirait à en imaginer un nouveau au niveau supranational. Ce système de couverture universelle replacerait les systèmes de base présents dans chaque État membre. Les contributions proviendraient d'un nouvel impôt européen spécialement créé et remplaçant une partie de celui perçu dans les systèmes nationaux (ou «beveridgiens ») et des cotisations, dans les systèmes professionnels (ou « bismarckiens »). Il serait rendu peu douloureux par son caractère substitutif, mais surtout par les contreparties patentes qu'il offrirait. Le Parlement européen se verrait confier la responsabilité du vote du montant de cet impôt chaque année. Dans ce nouveau système, le caractère décentralisé de certains systèmes nationaux (ou «beveridgiens ») serait repris, chaque État membre devenant une région. Pour des questions pratiques notamment de langue, la gestion administrative serait opérée par les administrations actuellement en place, mais 
qui seraient alors refondues dans une simple et même administration décentralisée. Par ailleurs, contrairement aux systèmes universels classiques, l'avance de frais serait retenue comme étant la règle, pour ses avantages en termes de responsabilisation des demandeurs de soins. Les patients couverts par les systèmes de couverture maladie universelle dans chaque pays seraient exonérés de cette avance de frais. La planification des soins demeurant de responsabilité nationale, les professionnels de la santé ne seraient pas salariés du système, mais recevraient certaines instructions de cette nouvelle institution (la Caisse d'assurance maladie européenne) qui publierait des recommandations contraignantes quant aux prescriptions notamment.

Déjà, les laudateurs et gestionnaires fulminent et crient au haro sur cet ambitieux projet? Soit. À l'impossible nul n'est néanmoins tenu, maxime qui devrait devenir l'antienne d'une nouvelle classe politique ambitieuse, n'ayant plus peur de choquer les responsables nationaux. Le nouveau paysage sanitaire européen inclura donc ce payeur supranational et les acteurs traditionnels, au premier rang desquels les régulateurs, devront adapter leurs interactions traditionnelles. Ces propositions supposeront une modification des traités et ne présentent qu'un modèle idéal, mais sans il semble incontournable de réfléchir à la future régulation de l'espace de santé européen.

\subsection{Création d'une Agence européenne d'évaluation des technologies de santé (EAHTA)}

Résolument axé sur la question de solidarité fondatrice d'un nouveau souffle en matière d'intégration européenne, cet article envisage ici un domaine crucial de l'action en santé publique. À l'heure actuelle, en effet, la durabilité des systèmes de santé européens est mise en cause, en raison notamment du coût croissant des nouvelles technologies. Or, la découverte de nouveaux besoins et de nouveaux traitements est constante. Étant donné la finitude des budgets de santé, une question se pose alors : comment une société donnée doit-elle répartir des dépenses de santé au niveau collectif ? Les priorités sont déterminées au niveau politique, suivant différents choix de société, qui doivent être présentés comme tels et assumés. Dans ce domaine, qui a trait aux budgets nationaux et à la solidarité intergénérationnelle, la valeur ajoutée de l'Union européenne peut s'avérer déterminante. C'est pourquoi, il est nécessaire de mettre sur pied une Agence européenne d'évaluation des technologies de santé (EAHTA). Conçue comme prenant place dans un ensemble cohérent avec la caisse d'assurance maladie européenne de base (envisagée ci-dessus), cette proposition permettrait de compléter le dispositif échafaudé au niveau supranational. Pour les produits médicinaux (médicaments), l'évaluation des technologies de santé constitue une sorte de phase d'expérimentation dans le milieu réel, une fois que le médicament a été autorisé sur le marché. Harmoniser l'évaluation scientifique des technologies de santé, autrement nommée Health Technology Assessment (HTA) constituerait certes une véritable révolution dans la prise de décision en matière de santé publique. Ceci permettrait surtout de penser ensemble la transformation de 
nos systèmes de soins européens et d'éviter le fractionnement des décisions dans vingtsept États-membres.

Comme l'indique la Haute autorité de santé française (HAS), « l'objectif [de l'évaluation des technologies de santé] est d'éclairer la décision publique par un avis argumenté prenant en compte les différentes dimensions du sujet». Autrement dit, il s'agit bien d'une présentation à visée scientifique dont l'unique objecif est de servir de guide à l'action politique. Il est sans doute nécessaire de rappeler que l'expression «technologies de santé » est ici entendue de manière très large. «Elle couvre les équipements, dispositifs médicaux, médicaments et procédures médicales ou chirurgicales utilisés en diagnostic, prévention, traitement et rééducation, ainsi que les systèmes organisationnels requis pour leur mise en œuvre. Il peut s'agir de technologies diffusées ou en voie de diffusion, ou de technologies émergentes ». L'évaluation se fait ainsi « sur des matériels, des équipements et des procédures ou stratégies utilisés par des professionnels de santé dans un but préventif, diagnostique ou thérapeutique » (Haute autorité de santé, site officiel, consultation le 13/07/09). Ce processus d'évaluation est pluridisciplinaire. Il vise à collecter l'information (dans les domaines médical, social, économique et éthique) liée à l'utilisation d'une technologie de santé d'une manière systématique, transparente, objective et robuste (Projet EUnetHTA, site officiel, consultation le 13/07/09).

« Grâce à la recherche et à l'innovation, de nouvelles technologies ayant le potentiel d'améliorer la santé des populations au travers de soins plus efficaces, sont continuellement introduites. [...] Cependant, tous les développements technologiques ne se traduisent pas par des gains sanitaires nets. L'histoire de la médecine et de la santé comptent de nombreux exemples de technologies qui n'ont pas produit les bénéficent escomptés ou qui se sont avérés nocifs. Néanmoins, les technologies qui ont prouvé leur efficacité - c'est-à-dire celles associées à améliorations sanitaires pertinentes - créent un défi constant pour les systèmes de santé dès lors que leur application peut requérir des ressources additionnelles (et pas seulement financières) ou la redistribution de ressources existantes limitées au sein d'un système de santé » (Garrido, Kristensen, Nielsen \& Busse, 2008, p. 1).

Les techniques d'évaluation des technologies de santé « consistent à simuler une situation où des prix reflétant la rareté des facteurs de production et les préférences sociales existeraient, et à les introduire dans des bilans chiffrés appelés coût-efficacité ou coûtavantages pour comparer des stratégies de santé, de prévention ou de soins et constituer des indicateurs d'aide à la décision. Comme escompté, « ces évaluations fournissent des critères de priorité au décideun» (Majnoni d'Intignano, B., 2001, p. 44). Ce sont ces approches qui aident à « définir le panier de services médicaux financés par les prélèvements obligatoires dans les pays européens. L'évaluation économique pose toutefois des problèmes théoriques et éthiques » (Ibid., p. 44-45). À première vue, « ces techniques d'évaluation peuvent choquer le monde médical. [C'est cependant ainsi que s'améliore] le processus de décision collectif en amont de la décision médicale individuelle » 
(Ibid., p. 46-47). Face à des budgets limités de chaque caisse nationale d'assurance maladie - et a fortiori de la future caisse européenne -, il est cependant nécessaire d'élaborer des stratégies de définition des priorités de remboursement qui ne laissent pas de place au hasard ou à l'arbitraire. Les évaluations de type HTA « rassemblent l'ensemble des paramètres médicaux, économiques et sociaux, et forment en général un dossier éclairant pour le décideur » (Ibid., p. 47). Parmi les questions auxquelles le HTA doit répondre figure celle qui suit. «Faut-il préférer une médicalisation généralisée à un coût élevé, mais avec un effet bénéfique sur la qualité de vie de large population, ou au contraire, une approche sélective, traitant les personnes à risque global élevé, à moindre coût, mais avec un effet plus limité » (Ibid., p. 48). L'Agence européenne d'évaluation des technologies de santé (EAHTA) aura alors pour tâche de fournir au décideur de la caisse d'assurance maladie européenne l'évaluation scientifique nécessaire à la prise de décision en matière de prix et remboursement.

Pour quelles raisons faudrait-il évaluer les technologies de santé au niveau européen ? Tout d'abord, et de manière assez prévisible, parce que les États-membres de l'UE ont mis en oeuvre des méthodes pour leur HTA relativement dissemblables. Il est donc nécessaire de rapprocher dès à présent les différentes pratiques dans ce domaine crucial et en développement, afin de poursuivre dans le procès de convergence des systèmes de santé européens. Deuxièmement, certains États-membres n'ont encore aucune expérience dans le domaine du HTA. Il serait opportun qu'ils développent leur expertise directement à partir d'un modèle consensuel et commun. Troisièmement, évaluer les technologies de santé au niveau européen permettrait un apprentissage mutuel en vue de la mise en place de ce modèle. Quatrièmement, cette évaluation supranationale aurait un sens, car nombre de " petits pays » n’ont pas forcément les ressources nécessaires pour mettre en place des évaluations systématiques, pour toutes les nouvelles technologies leur parvenant. Cinquièmement enfin, il s'agirait d'éviter la duplication des efforts, la perte de temps, afin de réduire les coûts dans ce domaine, par des économies d'échelle toutes supranationales.

\subsection{Création d'une Agence européenne de détermination des prix et des taux de remboursements}

Une fois l'évaluation scientifique des technologies de santé effectuée, les autorités publiques doivent donc décider quels traitement seront remboursés et à quels prix. La conséquence directe de la création d'une caisse d'assurance maladie européenne et d'une agence chargée de l'évaluation des technologies de santé est la nécessité de mettre en place un organisme dédié à l'élaboration des stratégies communes en matière de détermination des prix et des taux de remboursements au niveau européen. Bien entendu, les différences de niveaux de vie et de salaires varient fortement selon les vingt-sept États-membres. C'est pourquoi, cet organisme sera habilité, dans un premier temps, à déterminer différents prix selon les pays. À terme, il aura pour tâche de publier des prix 
uniformes sur le territoire de l'UE. C'est lui aussi qui dans sera chargé d'établir les taux de remboursement des traitements proposés. Il se basera pour se faire sur les avis de l'Agence européenne pour le HTA. À nouveau, il existe en ce domaine une valeur ajoutée certaine à regrouper les décisions au niveau européen. Plus que de se parler ou « d'échanger des bonnes pratiques », selon la formule consacrée, il s'agirait ici de réfléchir puis de décider ensemble, pour ensuite peser de manière crédible auprès des acteurs privés. Dans le contexte actuel de grande difficulté financière, décider d'une politique commune en matière de promotion des génériques éviterait par exemple la cacophonie et les expériences tâtonnantes de régulation de ce marché.

\subsection{Création d'une véritable « Direction générale de la santé publique »}

Dans le microcosme européen de Bruxelles, «DG» est le doux nom qui désigne l'équivalent des Ministères au niveau national. La Commission européenne se divise donc en Directions-Générales, dont le modèle ultra-structuré et hiérarchisé semble ne rien laisser au hasard. L'interlocuteur, plus que l'équivalent, des vingt-sept Ministères de la Santé, la Direction générale de la santé et des consommateurs (DG SANCO), est jeune. Elle va officiellement fêter ses dix ans cette année, en 2009: le 13 septembre 1999, l'ancienne DGXXIV (ou « Politique du consommateur et Protection de la santé du consommateur ») est devenue la DG SANCO. Il est à noter que cette DG, placée sous l'autorité du commissaire irlandais David Byrne, était la dernière née des vingtquatre directions générales existant à l'époque. L'acronyme a d'abord signifié «Santé et Protection des consommateurs» pour, depuis plus d'un an, conserver uniquement l'intitulé «Santé et Consommateurs ».

La création progressive d'une DG santé est à mettre en parallèle d'une communautarisation tardive de politiques publiques en la matière. Il est possible d'en faire remonter les prémices à 1965 (Directive sur les produits pharmaceutiques). La deuxième moitié des années 1980 voit une première légère inflation de réglementation. Le Conseil de l'UE dans sa formation santé commence à organiser des rencontres plus fréquentes, ce qui a une incidence sur le volume de législation en préparation : plan cancer (1987-89 et 1990-1994), "L’Europe contre le SIDA" (1991-93), plan de lutte contre la drogue (1990, 1998). Toutefois, à la suite de l'adoption d'une communication, par la Commission, portant sur des suggestions pour une politique communautaire de la santé publique, le Conseil de l'UE a quelque peu étouffé l'ambition de celle-ci, la forçant à se recentrer sur l'existant.

Comme souvent dans l'histoire de l'intégration européenne, cependant, les crises imprévues poussent les États-membres à se tourner vers l'Europe en quête de solutions. Celle de l'Encéphalopathie spongiforme bovine (ESB), autrement nommée de la «vache folle », en avril 1997, n'échappe pas à la règle. D’un avis assez généralement partagé, cette affaire «est incontestablement d'une gravité sans précédent et marque la fin de 
«l'âge d'or» de la Commission, celui où elle incarnait sans contestation possible l'intérêt européen. Encore aujourd'hui, elle n'a pas regagné sa légitimité » (Clarisse \& Quatremer, 2005, p. 91). Parmi les nombreux griefs faits à la Commission, il lui est notamment reproché d'avoir « délibérément caché la gravité de la crise de la vache folle, et ce dans l'intérêt des éleveurs et de l'industrie agroalimentaire» (Ibid.), ainsi que d'avoir trop souvent favorisé le marché comme valeur suprême, au détriment de la santé publique. Or, à partir de mars 1996, le lien entre l'ESB (chez l'animal) et la nouvelle variante de la maladie de Creutzfeldt Jakob (chez l'homme) commence à être fait. Dans ce contexte, la commission d'enquête parlementaire révélera une «campagne de désinformation » organisée par la Commission entre 1990 et 1995 (Ibid., p. 95).

A l'époque, la sécurité alimentaire et les aspects vétérinaires connexes étaient de la responsabilité de la DG VI «Agriculture ». Cependant, à la suite de la gestion calamiteuse de cette crise par la Commission et du véritable tollé qu'elle provoque à tous les étages de l'institution, ils vont lui être retirés. Une séparation claire des fonctions législatives, d'expertise scientifique et de contrôle est opérée. La future DG SANCO devient dépositaire d'une fonction législative vétérinaire ; elle est aussi responsable des comités scientifiques indépendants (en charge de l'évaluation scientifique des risques), précurseurs de l'Autorité européenne de sécurité des aliments (EFSA), dont la création remonte à janvier 2002. Parallèlement, un Office alimentaire et vétérinaire (FVO) est mis en place. Pour être complet, il faudrait enfin ajouter qu'avant 1999, il existait également une Direction de la santé publique et la sécurité au travail au sein de la DG EMPL (DG de l'Emploi et des affaires sociales). Ses «faits d'armes» dans le domaine sanitaire comprennent la mise en place de nombreuses directives établissant des prérequis sanitaires minimaux pour les divers et nombreux lieux de travail successivement ciblés. Le deuxième domaine sanitaire majeur dans lequel la DG Emploi agissait - et agit - est celui des services de santé au travers de la coordination des systèmes de sécurité sociale des employés et de leurs familles se déplaçant dans l'espace communautaire (règlements de 1971 et 1972). Ils méritent d'être mentionnés car la DG SANCO, en étendant progressivement son champ de compétence dans le domaine de la mobilité des patients, en viendra à empiéter sur ce domaine traditionnellement réservé à la DG EMPL.

Bien entendu, ce sont surtout les compétences communautaires dans le domaine de la sécurité alimentaire qui sortent renforcées de cette crise de l'ESB. Plus globalement néanmoins, l'importance de disposer de services administratifs dédiés aux questions de santé publique commence à s'imposer. Ainsi, depuis le traité d'Amsterdam (signature : le 2 octobre 1997; entrée en vigueur : le $1^{\text {er }}$ mai 1999), la Commission peut se saisir de la question de la qualité et de la sécurité des organes, et des substances d'origine humaine (le sang et ses dérivés, les tissus, les cellules), ainsi que des domaines vétérinaires et phytosanitaires, afin de présenter des normes contraignantes, soumises par la suite à la procédure de codécision. Pourtant, la DG SANCO qui s'échafaude penche bien vite de son côté « sécurité alimentaire ». À l’heure actuelle, la DG se subdivise en six directions, dont cinq opérationnelles. Trois d'entre elles sont liées à la sécurité des produits alimen- 
taires : Santé et bien-être animal (Direction «D $)$, Sécurité de la chaîne alimentaire ( $\mathrm{E} »)$ et l'Office vétérinaire ( $\mathrm{F} »)$, basé en Irlande. Mis à part la direction « B » en charge de la politique des consommateurs, qui dépend d'ailleurs d'un autre Commissaire que le reste de la DG depuis janvier 2007, il reste surtout une direction, la «C » («Santé publique et évaluation du risque », qui est subdivisée en sept unités), pour développer une politique communautaire de santé publique. Il faut bien évidemment tempérer ce jugement car il existe également une Agence exécutive pour la santé et les consommateurs (EAHC) $)^{14}$, chargée de mettre en place le programme de santé publique de l'Union européenne, ainsi qu'un Centre européen de prévention et de contrôle des maladies (ECDC), dont le but est de "contribuer à renforcer la protection de l'Europe contre les maladies infectieuses telles que la grippe, le SARS et le HIV/AIDS"15; son rôle de surveillance est particulièrement patent en ces temps de grippe $\mathrm{A}(\mathrm{H} 1 \mathrm{~N} 1)$.

Depuis ces dix dernières années, la DG SANCO s'est progressivement forgé une réputation et une expertise en matière de santé publique. Au regard des prérogatives des vingt-sept Ministères de la santé, ses capacités d'action semblent limitées. Deux raisons peuvent notamment être invoquées. Ceci est dû tout d'abord au caractère spécifique de la santé, qui a trait aux prérogatives redistributives des États providences. La santé est centrale dans le budget des États-membres : 8.5\% en moyenne, en 2002 (Husson, 2004, p.134). Les dépenses occasionnées par le remboursement et la planification des soins notamment les conduisent à une extrême prudence lors des débats au niveau supranational. D'autre part, au niveau européen, l'action en matière de santé est éclatée entre plusieurs directions-générales. Ainsi, la DG Entreprises et industrie est compétente pour utiliser l'article 152 et dispose de trois unités intervenant dans le domaine de la santé : la «F-2 produits pharmaceutiques », la «F-3: Cosmétiques et dispositifs médicaux » et la «F5: Compétitivité de l'industrie pharmaceutique et des biotechnologies». La DG Société de l'information et médias possède une unité chargée des nouvelles technologies de l'information et de la communication en matière de santé. Elle s'occupe entre autres des questions de télémédecine et de santé en ligne (" ehealth»). L'une des quinze directions de la DG de la recherche porte également l'intitulé «santé » et les fonctionnaires qui y servent sont chargés de mettre en œuvre le septième programmecadre de recherche et de développement (PCRD) technologique pour la période 20072013. De nombreux projets de recherche dans le domaine de la santé sont financés et bénéficient d'une partie des 53,2 milliards d'euros disponibles (sur sept ans) à travers ce PCRD. La DG Emploi et affaires sociales Santé, quant à elle, traite de questions sanitaires au sein de l'unité « Sécurité et hygiène au travail» (F-4), mais également dans la direction «Protection et intégration sociales » (E), où une unité «Coordination des régi-

\footnotetext{
${ }^{14}$ http://ec.europa.eu/eahc/index.html

$15 \mathrm{http} / /$ /europa.eu/agencies/community_agencies/ecdc/index_fr.htm
} 
mes de sécurité sociale, libre circulation des travailleurs » est notamment chargée de faire fonctionner le règlement $n^{\circ} 1408 / 71$ précité. La DG EMPL gère également le Fonds social européen, au travers duquel sont financées certaines actions de formation pour les professionnels de santé. Enfin, la DG REGIO est en charge du Fonds européen de développement régional (FEDER), qui sert notamment pour le cofinancement de projets en matière d'infrastructures sanitaires.

Cet éclatement de l'action communautaire en matière de santé est souvent idoine et justifié, mais, depuis quelques temps, certaines voix s'élèvent en faveur d'un rapatriement d'une partie de ces services, alors englobés dans un organigramme cohérent. Après avoir renforcé l'Agence des médicaments; après avoir mis sur pied une caisse d'assurance maladie européenne de base et deux organismes chargés de l'évaluation des technologies de santé, pour l'une, et de détermination des prix et des taux de remboursement des traitements, il serait, en effet, imaginable qu'une Direction générale Santé prenne place dans ce nouveau dispositif. Elle regrouperait notamment les services en charge de la régulation des médicaments et des dispositifs médicaux de la DG ENTR. Nombreux sont les appels - provenant notamment d'interlocuteurs fréquents de la DG SANCO, tels EPHA, AIM et BEUC, tous trois signataires d'une lettre ouverte en ce sens : "Governance change needed: EU pharmaceutical policy should be the responsibility of a health focussed directorate within the European Commission", le 17 janvier 2009 - à donner un nouvel élan à la DG SANCO actuelle et à lui faire franchir une étape ambitieuse. Alors, après avoir rassemblé en un seul lieu - Bruxelles - les services éclatés entre Luxembourg et Bruxelles (pour la Direction « $C »)$, il serait possible d'envisager des politiques de santé publique au niveau européen encore plus ambitieuses. Les sujets pour une intervention utile du niveau supranational ne manquent d'ailleurs pas. Le travail pourrait se poursuivre dans les domaines suivants : déterminants de santé, lutte contre le cancer, qualité et sécurité des soins, gestion des flux de professionnels de santé, organisation de la prévention des maladies au niveau européen, gestion de la lutte contre les pandémies de type AH1N1. Seulement, pour que cette DG Santé n'ait pas l'impression de faire de la figuration face à des États-membres récalcitrants, il lui faudrait sans doute aussi envisager d'étendre les bases juridiques sur lesquelles elle s'appuie.

\subsection{Extension des compétences communautaires en matière de santé}

Pour l'heure, la seule partie des traités communautaires traitant explicitement de santé publique est le sommaire article $152 \mathrm{CE}$. Il existe cependant deux voies communautaires possibles d'intervention en matière de santé publique : soit sur la base de cet Art. $152 \mathrm{CE}$, qui ne prévoit pas d'harmonisation et laisse des marges de manœuvre limitées; soit sur la base de l'Art. $95 \mathrm{CE}$, avec une vraie compétence d'harmonisation (ex: législation en matière de tabac), la santé étant alors considérée en tant qu'accessoire indispensable au bon fonctionnement du marché intérieur. Créer une DG ambitieuse né- 
cessite de la doter de véritables compétences. Cependant, si la base juridique prévue par le Traité CE pour la santé est très rudimentaire et restreint les velléités d'action dans le domaine, d'autres possibilités existent. La directive « relative à l'application des droits des patients en matière de soins de santé transfrontaliers » (Proposition de directive en cours d'adoption, adoptée par la Commission européenne le 2 juillet 2008), en est un bon exemple. Le texte fait donc référence à l'article $95 \mathrm{CE}$, selon lequel « Le [...] Conseil arrête les mesures relatives au rapprochement des dispositions législatives, réglementaires et administratives des États-membres qui ont pour objet l'établissement et le fonctionnement du marché intérieur. [...] La Commission, [...] en matière de santé, de sécurité, de protection de l'environnement et de protection des consommateurs, prend pour base un niveau de protection élevé en tenant compte notamment de toute nouvelle évolution basée sur des faits scientifiques » (Traité instituant la Communauté européenne, version consolidée de 2006). Craignant de voir la Commission s'engouffrer trop avant dans une brèche, celle de l'harmonisation dans le secteur de la santé, les États-membres en rappellent les spécificités et insistent pour que la base juridique utilisée soit l'article 152. En effet, ce dernier établit que :

"L'action de la Communauté, qui complète les politiques nationales, porte sur l'amélioration de la santé publique et la prévention des maladies et des affections humaines et des causes de danger pour la santé humaine. Cette action comprend également la lutte contre les grands fléaux, en favorisant la recherche sur leurs causes, leur transmission et leur prévention ainsi que l'information et l'éducation en matière de santé. [...] La Communauté encourage la coopération entre les Étatsmembres dans les domaines visés au présent article et, si nécessaire, elle appuie leur action. [...] L'action de la Communauté dans le domaine de la santé publique respecte pleinement les responsabilités des États-membres en matière d'organisation et de fourniture de services de santé et de soins médicaux » (Ibid.).

Cet encadrement de l'action sanitaire communautaire ne permet pas d'adopter des textes de loi ambitieux, tout simplement parce que les États-membres ne le souhaitent pas et tentent de conserver leurs prérogatives. Certains semblent être d'avis que favoriser une action par le truchement de cet article amplifie la mécanique européenne d'intervention par le marché. Ils refusent d'adosser le chapitre concernant la coopération entre États-membres en matière de santé à l'article $95 \mathrm{CE}$. Il s'agit selon eux d'éviter : de donner, tout d'abord, une compétence d'harmonisation à la Commission, ce qui est impossible dans le cadre de l'Art. $152 \mathrm{CE}$; de permettre ensuite l'adoption de mesures législatives trop contraignantes (notamment de type réglementaire); de favoriser enfin la mise en place de mesures d'exécution (comitologie). À ce dernier stade, la comitologie peut en effet être gênante si elle permet à la Commission d'avoir le dernier mot en cas d'opposition sur le contenu des mesures avec le Conseil. La comitologie est le terme technique désignant la phase d'exécution d'un texte juridique communautaire (en comités). Les États-membres y délèguent à la Commission leur pouvoir d'exécution. « Fo- 
rums de discussion, les comités sont composés de représentants des États-membres et présidés par la Commission. Ils permettent à la Commission d'instaurer un dialogue avec les administrations nationales avant d'adopter des mesures d'exécution. La Commission s'assure ainsi qu'elles correspondent au mieux à la réalité de chaque pays concerné » (Site officiel Europa, glossaire). Le domaine des services de santé qui se retrouve en toile de fond de cette directive est sensible pour les États-membres car il touche à leurs capacités d'organisation et de planification des soins sur leurs territoires.

Les deux seuls domaines sanitaires où l'Union peut adopter des normes juridiquement contraignantes sont celui des médicaments, ainsi que celui des dispositifs médicaux, dans lequel il semble difficile d'envisager davantage d'harmonisation pour le moment. Ses marges de manœuvre sont faibles.

\begin{abstract}
«L'Union n'est en effet autorisée qu'à établir des mesures d'encouragement pour traiter ces enjeux de santé publique. Il lui est interdit d'adopter des mesures visant à harmoniser les dispositions législatives et réglementaires des États-membres. [...] En interdisant a priori à l'Union l'harmonisation des dispositions nationales, on se prive d'instruments qui peuvent, fût-ce à titre exceptionnel, apporter un appui d'envergure aux actions entreprises au niveau national afin de protéger la santé publique. Certaines décisions sont en effet plus faciles à faire adopter au niveau européen qu'au niveau national. Le meilleur exemple est sans doute celui de la législation communautaire interdisant pour partie la publicité du tabac et réglementant l'étiquetage des produits du tabac et leur contenu. Le niveau européen a permis de contourner les puissants intérêts économiques qui bloquaient l'adoption de telles mesures dans plusieurs États-membres. Faute de base juridique adéquate, l'Union a toutefois du détourner ostensiblement les traités et construire cette législation sur une base juridique destinée à assurer le bon fonctionnement du marché intérieur, l'article 95 du traité, ce qui l'a par exemple empêchée d'interdire purement et simplement toute forme de publicité des produits du tabac » (Guigner, 2008, p. 4344).
\end{abstract}

La situation actuelle n'est pas idéale. Il est par conséquent nécessaire de modifier les bases juridiques des traités, ne serait-ce que pour y faire figurer les propositions de ce plaidoyer utopiste. Il convient d'adopter un volontarisme apte à porter le débat auprès des responsables nationaux, qui seuls peuvent le traduire en changements manifestes.

\title{
Conclusion
}

L'organisation de la solidarité et, partant, des systèmes nationaux de santé, par les États-providence varie considérablement d'un pays européen à un autre. En Europe, deux grands modèles d'organisation de ces systèmes de santé existent: le «beveridgien » et le « bismarckien ». Si on garde présent à l'esprit l'importance du cadre national de l'État-providence dans la régulation du champ de la solidarité, il n'est pas surprenant 
que les États-membres de l'UE considèrent que régulation, financement et prestation des soins de santé soient essentiellement des prérogatives nationales. Mais depuis les années 1990, les systèmes de santé sont devenus de plus en plus complexes dans leurs structures, suite à de multiples réformes. Réduction des coûts, tickets modérateurs pour certains médicaments ou traitements, listes d'attentes pour certaines opérations, sont les conséquences visibles de ces modifications de grande ampleur. Dans certains cas, il est donc devenu plus «attrayant» pour un patient de profiter de l'offre d'un prestataire de soins d'un autre État-membre européen. S'il peut paraître éloigné des préoccupations du grand public, ce puissant phénomène qu'est la mobilité des patients met en réalité en question la régulation nationale des systèmes de santé. C'est par cette porte entrouverte que peut s'engouffrer l'Europe de santé du XXIe siècle. C'est ainsi que, mus par une réflexion utopiste, nous avons imaginé une Europe de la santé idéale, qui passe par la création d'une assurance maladie universelle de base européenne assurant le remboursement des soins primaires et financée par un impôt européen. Cette assurance est alors complétée par une agence européenne d'évaluation des technologies de santé et par une agence européenne de détermination des prix et des taux de remboursements.

Gageons donc que la grande innovation de l'UE de ce début de siècle peut être la mise en œuvre d'un système de santé fonctionnant pour 500 millions d'Européens. L'objectif ici n'est pas de rechercher la recréation d'un super État-providence européen et de simplement transposer le cadre national au niveau supranational avec pour seul but de penser dans les étroites limites de l'existant. Il consiste bien plutôt à imaginer le cadre collectif européen qui permettrait d'organiser le principe de solidarité et de le rendre tangible. Bien sûr, cela pousse vers davantage d'intégration politique, mais c'est uniquement en se lançant dans cette ambitieuse aventure que l'on saura si l'Union de ces puissants États-nations peut fonctionner. C'est l'idée généreuse de femmes et d'hommes qui croient fermement que c'est dans le projet d'intégration européenne que s'incarnent le mieux les valeurs d'humanistes nées sur son territoire.

Certains seraient alors tentés de s'indigner. "Mais que reste-t-il au national et au régional ? Votre projet "ne tient pas la route", les États-membres ne voudront pas se départir de leurs prérogatives. Ils n'auront de cesse d'annihiler vos plans. Vous voici prévenus! » A tous ceux-ci, nous souhaitons rappeler que nous avons voulu ici mêler une approche didactique, analytique et descriptive à la fois, mais qu'à cette dimension académique, s'ajoute ici une réflexion utopiste, car le rêve européen s'effrite. «On ne fait pas rêver les gens avec un marché unique! », disait en substance Jacques Delors. On ne les fait pas non plus rêver avec une Europe en friche et inachevée. Le point de vue défendu ici est que le projet européen doit retrouver un nouveau souffle et celui-ci ne peut passer que par un surcroît de solidarité. Tout n'a pas été fait ni tenté au niveau européen pour organiser ce grand espace de solidarité. Loin de vouloir recréer un super État dominant cinq cents millions de citoyens fort divers, il nous semble souhaitable d'organiser un système redistributif au niveau supranational. Ceci permettrait de répondre tous ensemble aux défis de l'époque moderne. 


\section{Bibliographie}

\section{Textes officiels :}

Arrêt CJCE du 23 octobre 2003, Inizan, Aff. C-56/01, Rec. p. I-12403.

Arrêt CJCE du 12 juillet 2001, Vanbraekel, Aff. C-368/98, Rec. p. I-05363.

Arrêt CJCE du 28 avril 1998, Kobll, Aff. C-158/96, Rec. p. I-01931.

Arrêt CJCE du 12 juillet 2001, Smits-Peerbooms, Aff. C-157/99

Arrêt CJCE du 13 mai 2003, Müller-Fauré, Aff. C-385/99. Rec. p. I04509.

Arrêt CJCE du 16 mai 2006, Watts, Aff. C-372/04.

Versions consolidées du Traité sur L'Union européenne et du Traité Instituant la Communauté européenne (version consolidée), Journal officiel $n^{\circ}$ C $321 E d u 29$ décembre 2006 :

http://eur-

lex.europa.eu/LexUriServ/LexUriServ.do?uri=OJ:C:2006:321E:0001:0331:FR:pdf, consultation le jeudi 06 mars 2008.

Règlement (CEE) No 1408/71 du Conseil, relatif à l'application des régimes de sécurité sociale aux travailleurs salariés, aux travailleurs non salariés et aux membres de leur famille qui se déplacent à l'intérieur de la Communauté, 14 juin 1971, (JO L 149 du 5.7.1971, p. 2), (Version consolidée - JO noL 28 du 30. 1. 1997, p. 1).

Conseil de l'Union européenne, Communiqué de presse, $2733^{e ̀ m e}$ session du Conseil Emploi, politique sociale, santé et consommateurs, Luxembourg, les 1er et 2 juin 2006, p. 33-37.

Sénat français, Rapport d'information sur l'Union européenne et les services de santé, Roland Ries, rapporteur au nom de la délégation pour l'Union européenne, Rapport $\mathrm{N}^{\circ} 186,30$ janvier 2007, 38 pages, p. 6.

Directive 65/65/CEE du Conseil, du 26 janvier 1965, concernant le rapprochement des dispositions législatives, réglementaires et administratives, relatives aux spécialités pharmaceutiques, JO nº 022 du 09/02/1965 p. 0369 - 0373.

Proposition de directive du Parlement européen et du Conseil relative à l'application des droits des patients en matière de soins de santé transfrontaliers, adoptée par la Commission européenne le 2 juillet 2008 :

http://ec.europa.eu/health/ph_overview/co_operation/healthcare/docs/COM_fr.pdf 


\section{Articles et livres :}

BAETEN R., «L'impact potentiel sur les systèmes de soins de santé de la proposition de directive sur les services », Observatoire Social Européen, Notabene 135, October 2004.

BAETEN, R. \& GLINOS, I. A. 2006. À Literature Review of Cross-Border Patient Mobility in the European Union. EUROPE FOR PATIENTS, ED. Brussels.

CASSAN M., L'Europe communautaire de la santé, Université Aix-Marseille III, Centre d'études et de recherches internationales et communautaires, Economica, 1989, 274 pages.

CLARISSE Y. \& QUATREMER J., Les maîtres de l'Europe, Grasset, 2005, 409 pages.

DUBOUIS L. \& BLUMANN C., Droit matériel de l'Union européenne, Montchrestien, Domat droit public, 2004. 3ème edition, 676 pages.

ESPING-ANDERSEN, G. 1998. The three worlds of welfare capitalism. Repr. Princeton, N.J: Princeton Univ. Press.

FERRERA, M. 2006. The boundaries of welfare. European integration and the new spatial politics of social protection. Reprinted. Oxford: Oxford University Press.

GARRIDO, KRISTENSEN, NIELSEN \& BUSSE, Health Technology Assessment in Europe and health policy-making in Europe, Current status, challenges and potential, European Observatory on Health Systems and Policies, Observatory Studies Series, $\mathrm{N}^{\circ} 14,2008,181$ pages.

GUIGNER S., La santé : un enjeu vital pour l'Europe, Fondation Notre Europe, Etudes et recherches, $\mathrm{N}^{\circ}$ 68, décembre 2008.

HARVEY, T. K. \& MCHALE, J. V. 2004. Health Law and the European Union. Cambridge: Cambridge University Press.

HATZOPOUlOS, V. G. Current Problems of Social Europe. COLLEGE OF EUROPE, ED. Bruges. (Research Papers in Law). [WWW] www.coleurop.be. (21 January 2008).

HUSSON M., La santé, un bien supérieur?, Chroniques Internationales de l'IRES - n 91 , novembre 2004, pp. 134-150.

KACZOROWSKA, A. 2006. A Review of the Creation by the European Court of Justice of the Right to Effective and Speedy Medical Treatment and its Outcomes. European Law Journal, 12 (3), pp. 345-370.

KOSTERA T., Europeanizing Healthcare, Cross-border Patient Mobility and Its Consequences for the German and Danish Healthcare Systems, Bruges Political Research Papers, No 7, Mai 2008. 
LAMPING, W. 2005. European integration and health policy. À peculiar relationship. In: STEFFEN, Monika, ed. Health governance in Europe. Issues, challenges, and theories. London, New York, NY: Routledge. (Routledge/ECPR studies in European political science; 40), pp. 18-48.

MAJNONI D’INTIGNANO, B., Santé et économie en Europe, PUF, Que sais-je ?, 2001, 127 pages.

MERKEL B. et HÜBEL M., Public Health in the European Community. In: HOLLAND W. et MOSSALIOS E., Public Health Policies in the European Union, Ashgate, pp. 49-67, 1999.

MORDELET P., Gouvernance de l'hôpital et crise des systèmes de santé, Editions de l'Ecole Nationale de la Santé Publique, 2006, 367 pages,

POLTON D., "Les systèmes de santé dans les pays occidentaux », in TRONQUOY P. (dir.), La santé, La Documentation française, Cahiers français, $\mathrm{N}^{\circ} 324,2005,95$ pages.

PRADALIE N., Les services de santé, des services comme les autres?, Mémoire du Collège d'Europe, Mai 2008.

ROTHGANG, H. et al. 2008. The Changing Publi-Private Mix in OECD Health-care Systems. In: SEELEIB-KAISER, Martin, ed. Welfare state transformations. Comparative perspectives. Basingstoke: Palgrave Macmillan, pp. 132-146.

SEELEIB-KAISER, M., ed. 2008. Welfare state transformations. Comparative perspectives. Basingstoke: Palgrave Macmillan.

SIEVEKING, K. 2007. ECJ Rulings on Health Care Services and Their Effects on the Freedom of Cross-Border Patient Mobility in the EU. European Journal of Migration and Law, 9, pp. 25-51.

SINDBJERG MARTINSEN, D. 2007. EU for the Patients. Developments, Impacts, Challenges. SWEDISH INSTITUTE FOR EUROPEAN POLICY STUDIES, ED. Stockholm. (6). [WWW] http://www.sieps.se/publikationer/rapporter/eu-for-thepatients-developments-impacts-challenges.html. (3 May 2009).

SIMON A-C., "La mobilité des patients en droit européen», in NIHOUL P. \& SIMON A-C., L'Europe et les soins de santé, L.G.D.J \& Larcier, 2005, 405 pages, pp. $145-188$, p. 173

SPICKER, P. 2000. The welfare state. À general theory. London, Thousand Oaks, Calif: Sage Publications. 
STEFFEN, M., ed. 2005. Health governance in Europe. Issues, challenges, and theories. Routledge/ECPR studies in European political science, 40. London, New York, NY: Routledge.

STEFFEN, M., LAMPING, W. \& LEHTO, J. 2005. Introduction. The Europeanization of health policies. In: STEFFEN, Monika, ed. Health governance in Europe. Issues, challenges, and theories. London, New York, NY: Routledge. (Routledge/ECPR studies in European political science; 40), pp. 1-18.

\section{Sites internet :}

Projet EUnetHTA :

http://www.eunethta.net/HTA/

Haute Autorité de Santé :

http://www.has-sante.fr/portail/jcms/j_5/accueil

Site officiel Europa, glossaire :

http://europa.eu/scadplus/glossary/comitology_fr.htm 